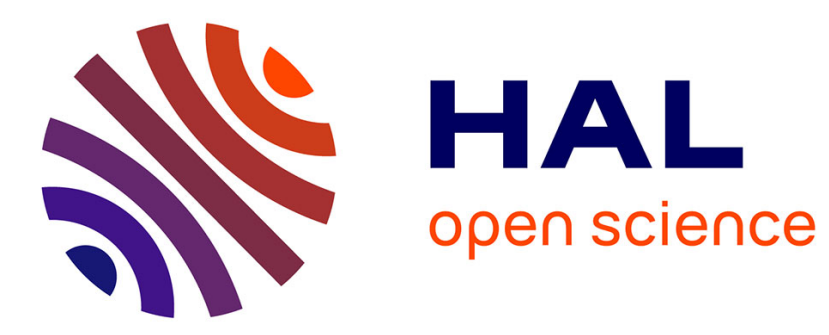

\title{
Location of distribution centers in a multi-period collaborative distribution network
}

Xin Tang, Fabien Lehuédé, Olivier Péton

\section{To cite this version:}

Xin Tang, Fabien Lehuédé, Olivier Péton. Location of distribution centers in a multi-period collaborative distribution network. INOC2015, May 2015, Varsovie, France. hal-01169614

\section{HAL Id: hal-01169614 \\ https://hal.science/hal-01169614}

Submitted on 29 Jun 2015

HAL is a multi-disciplinary open access archive for the deposit and dissemination of scientific research documents, whether they are published or not. The documents may come from teaching and research institutions in France or abroad, or from public or private research centers.
L'archive ouverte pluridisciplinaire HAL, est destinée au dépôt et à la diffusion de documents scientifiques de niveau recherche, publiés ou non, émanant des établissements d'enseignement et de recherche français ou étrangers, des laboratoires publics ou privés. 


\title{
Location of distribution centers in a multi-period collaborative distribution network
}

\author{
Xin Tang, Fabien Lehuédé, Olivier Péton ${ }^{1}$ \\ École des Mines de Nantes, Institut de Recherche en Communications et \\ Cybernétique de Nantes (IRCCyN, UMR CNRS 6597) \\ 4 rue Alfred Kastler, 44300 Nantes, France
}

\begin{abstract}
This paper presents a research study which aims at determining optimal locations of regional distribution centers in a collaborative distribution network. We consider a multi-layered distribution system between a cluster of suppliers from a given region and several thousands customers spread over the whole country. The optimization problem consists of finding the locations of intermediate logistics facilities called regional distribution centers and assigning customers to these facilities according to one year of historical data. The distribution system combines full truckload (FTL) routes and less-than-truckload (LTL) shipments. The use of several rates for transportation, as well as the high impact of seasonality implies that, for each shipping date, the number of FTL routes and the cost of LTL shipments should be precisely evaluated. This problem is modeled as a mixed integer linear problem and used as a decision aiding tool on a real case study related to the distribution of horticultural products in France.
\end{abstract}

Keywords: Collaborative distribution network, location-allocation

$\overline{1 \text { Email: }}\{$ xin.tang, fabien.lehuede, olivier.peton\}@mines-nantes.fr 


\section{Introduction}

Horizontal collaboration is defined as a business agreement between manufacturers at the same level in the supply chain in order to achieve a common objective. This may be realized by proper manipulation, utilization and sharing of appropriate resources [1]. However, companies which resort to horizontal collaboration are often competitors and they have to overcome many cultural, organizational and technical barriers. Competing companies are often reluctant to share strategic information or resources concerning their core business, while external operations such as the supply or distribution of goods are often natural candidates for collaboration.

The design of collaborative distribution networks belongs to the domain of supply chain network design. One key issue when designing a supply chain network is facility location, which has received considerable attention from academics and practitioners over the last several decades [3]. In general, facility location problems deal with the determination of the optimal number, capacity, type, and geographic location of facilities in such a way that the network cost is minimized while customer demand is satisfied. Facility location is often considered over a strategic planning horizon, generally at least several years.

In this paper, we focus on a cluster of competing companies in the same geographical area in Western France, which decide to establish horizontal collaboration for the delivery of their goods to a large set of customers spread over the whole country.

\section{Problem formulation}

\subsection{Facilities}

The distribution system uses a multi-layered distribution network, represented in Figure 1. It is composed of three types of facilities:

- Production Zone (PZ): composed of a set of suppliers and one Consolidation and Distribution Center (CDC). Suppliers are collaborating companies and they are the sources of all material flow in the network. The CDC acts as the main collaborative warehouse at the suppliers gate.

- A set of Regional Distribution Centers (RDCs) which are articulation points between FTL routes and LTL shipments in the collaborative network.

- A large set of customers that are the destinations of all product flows. 
Customer orders are firstly consolidated at the CDC by so-called supplier routes, and then delivered to the customers generally through a sequence of two successive transportation segments: FTL routes and LTL shipments. The main goal of this study is to locate RDCs. Then the set of all suppliers and the CDC can be aggregated into a single artificial facility PZ. PZ can be considered as a particular Distribution Center (DC) and is the single origin for all FTL routes (We have $D C s=R D C s \cup P Z$ ).

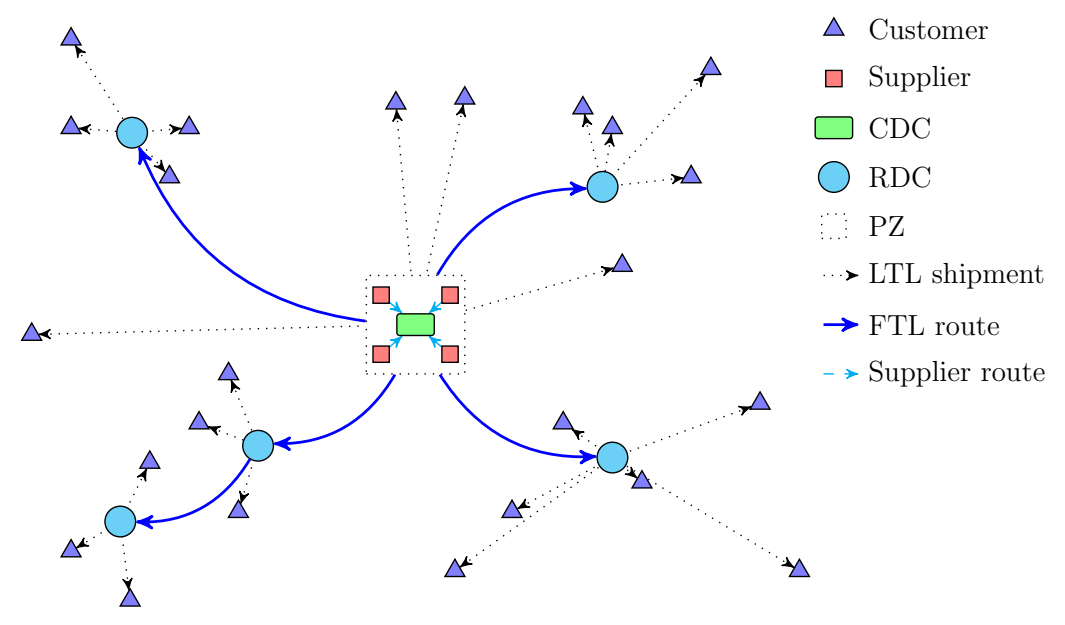

Figure 1. The collaborative distribution network

\subsection{FTL routes and shipments}

Due to shipments consolidation at PZ, FTL routes can be assumed between PZ and each RDC. The use of FTL routes implicitly assumes that enough shipment have been consolidated, making it possible to fill a full truckload vehicle. They can be a direct trip from PZ to one RDC or include several RDCs. In the latter case, stopover costs are charged. But in practice, multiple stopovers are not allowed due to time and quality of service constraints. In this study, one intermediate RDC is authorized to be used on FTL routes. The cost of an FTL route is proportional to the distance traveled or hours worked (wages of drivers, fuel, use of truck). It also integrates fixed costs such as tolls or stopover charges. The set of all FTL routes can be easily enumerated, with a good cost estimation which is independent of the number of units carried.

LTL shipments generally concern the distribution from RDCs or the PZ to final customers. These shipments are operated by local logistics service providers (LSP) and are not controlled by suppliers. We empirically observed that the cost of LTL shipments follows a modified all-unit discount (MAUD) 


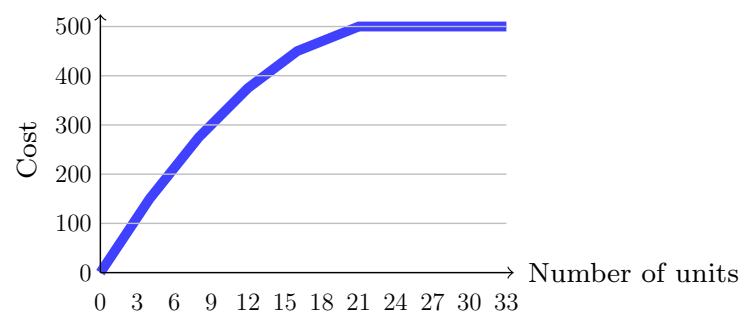

Figure 2. Cost of an LTL shipment

cost structure which is based on weight segments and leads to a non continuous piecewise affine cost curve with breakpoints. In order to estimate realistic LTL shipments cost (per unit), we followed the approach of le Blanc et al. [2], and estimated the cost by means of a regression model. The LTL cost could be expressed with good accuracy as a function of the number of units and the distance between the origin and the destination. Figure 2 illustrates an example of LTL cost from a given DC to a given destination and shows that the cost increases with the number of units transported and incorporates discount rates. This cost already includes handling costs at RDCs.

\subsection{Customer orders and departments}

We have several motivations not to aggregate customer demand over the time horizon and thus to cast this problem as a classical fixed charge facility location problem. First, a majority of customers order small quantities and shipment volumes are highly seasonal. During the high season, shipments size and frequency is much larger which reinforces the opportunity for using FTL routes than during the low season. Second, the cost of a given FTL route is a piecewise linear and discontinuous function, with each piece representing the fixed cost for using $n$ trucks ( $n$ times the cost of using one truck on this FTL route). Then we define three seasons according to the shipment volumes and we partition the whole set of customer orders into independent shipping dates. All customer orders are synchronized in a single shipment per date.

Each customer is delivered from the same DC during the whole time horizon. The territory is partitioned into administrative areas called departments. All customers in a department must be assigned to the same DC during the whole time horizon considered. Moreover, all customers in a department have the same LTL shipment cost function. Note that shipments from the PZ are authorized even for departments that are assigned to an RDC. DCs are crossdocking facilities only, all products enter and leave these facilities in the same 
shipping date. Therefore, our study does not include inventory planning for DCs.

The objective of our study is to design a collaborative distribution network composed of one PZ and several RDCs by determining the optimal number and location of RDCs, the allocation of departments to RDCs, the number of vehicles on FTL routes for each shipping date, while minimizing the sum of facility location cost and transportation cost.

\section{MILP formulation}

Let $J$ be the set of DCs. In order to model direct distribution from PZ to customers, PZ is defined as $j_{0}$. Hence, the set of RDCs is denoted by $J^{\star}=$ $J \backslash\left\{j_{0}\right\}$. We denote by $I$ the set of customers and $D$ the set of departments. For each $d \in D$, the set of customers located in department $d \in D$ is denoted by $I_{d}$. The set of departments that can be served from $j \in J$ is denoted as $D_{j}$. All departments can be served from the CDC, that is $D_{j_{0}}=D$.

The set of FTL routes is denoted by $\Omega$. We introduce $\Omega_{j}$ and $J_{\omega} \subseteq J^{\star}$ to denote the subset of FTL routes visiting $j \in J^{\star}$ and the subset of RDCs visited by route $\omega \in \Omega$, respectively. An FTL route $\omega \in \Omega$ is operated by a homogeneous fleet of vehicles of capacity $Q_{\omega}$.

We consider a set of shipping dates $T$ in a whole time horizon (one year). For each shipping date $t \in T$, the customers demand is known: $q_{i}^{t}$ represents the demand of customer $i \in I$ on date $t \in T$; $c_{i j}^{t}$ denotes the cost of LTL distribution of one unit of demand $q_{i}^{t}$ from $j \in J$ to $i \in I$. The fixed cost of running an RDC $j \in J^{\star}$ during the whole time horizon is denoted by $c_{j}$. Since the RDCs are operated by LSPs, it is assumed that they are already operating. Thus no fixed opening cost is associated with each RDC $j\left(c_{j}=0\right)$ but a variable cost is incurred as soon as the first unit is processed by RDC. We denote by $p$ the number of RDCs to be opened. The capacity of DCs is assumed to be infinite. Finally, we define the following decision variables including three sets of binary or integer variables:

- $y_{j}=1$ if RDC $j \in J^{\star}$ is opened, and 0 otherwise;

- $x_{d j}=1$ if department $d \in D$ is served by DC $j \in J$, and 0 otherwise;

- $n_{\omega}^{t} \in \mathbb{N}$, number of FTL vehicles used on route $\omega \in \Omega$ on date $t \in T$.

Two sets of continuous non-negative variables represent the material flow through the network:

- $u_{\omega j}^{t}$, number of units on route $\omega \in \Omega$ unloaded at RDC $j \in J^{*}$ on date 
$t \in T$

- $f_{i j}^{t}$, number of units shipped from DC $j \in J$ to customer $i \in I$, on date $t \in T$.

The location-allocation problem just described can be modeled as follows:

$$
\min z=\sum_{j \in J^{*}} c_{j} y_{j}+\sum_{t \in T} \sum_{\omega \in \Omega} c_{\omega} n_{\omega}^{t}+\sum_{t \in T} \sum_{i \in I} \sum_{j \in J} c_{i j}^{t} f_{i j}^{t}
$$

s.t.

$$
\begin{array}{ll}
\sum_{j \in J} x_{d j}=1 & \forall d \in D \\
\sum_{j \in J} f_{i j}^{t}=q_{i}^{t} & \forall i \in I, \forall t \in T \\
f_{i j}^{t} \leq q_{i}^{t} x_{d j} & \forall i \in I_{d}, \forall d \in D_{j}, \forall j \in J^{*}, \forall t \in T \\
\sum_{j \in J^{*}} y_{j}=p & \\
x_{d j} \leq y_{j} & \forall d \in D_{j}, \forall j \in J^{*} \\
n_{\omega}^{t} \leq y_{j} M_{j, \omega}^{t} & \forall \omega \in \Omega_{j}, \forall j \in J^{*}, t \in T \\
\sum_{i \in I_{d}, d \in D_{j}} f_{i j}^{t}=\sum_{\omega \in \Omega_{j}} u_{\omega j}^{t} & \forall j \in J^{*}, \forall t \in T \\
\sum_{j \in J_{\omega}} u_{\omega j}^{t} \leq Q_{\omega} n_{\omega}^{t} & \forall \omega \in \Omega, \forall t \in T \\
x_{d j} \in\{0,1\} & \forall d \in D, \forall j \in J \\
y_{j} \in\{0,1\} & \forall j \in J^{*} \\
n_{\omega}^{t} \in \mathbb{N} & \forall \omega \in \Omega, \forall t \in T \\
f_{i j}^{t} \in \mathbb{R} & \forall i \in I, \forall j \in J, \forall t \in T \\
u_{\omega j}^{t} \in \mathbb{R} & \forall \omega \in \Omega, \forall j \in J, \forall t \in T
\end{array}
$$

The objective function (1) sums up the fixed cost of opening facilities, the cost of FTL routes and the LTL distribution costs. Constraints (2) are single assignment constraints for each department. Constraints (3) ensure the satisfaction of customer demand. Constraints (4) state that if RDC $j \in J^{*}$ does not serve department $d \in D_{j}$, then all flows from $j$ to the customers $i \in I_{d}$ must be 0 . Constraint (5) indicates the number of facilities to be opened which is defined by suppliers. Constraints (6) state that a department $d$ can only be allocated to by an opened RDC. Since $j_{0}$ is opened, this constraint applies 
only for $j \in J^{*}$. Constraints (7) state that if an RDC is closed, then the FTL routes visiting this location are not operating. Constraints (8) model flow conservation at an RDC $j \in J^{\star}$. Constraints (9) model vehicles capacity. Constraints (10)-(14) define the nature of decision variables.

For Constraints (7), a possible way to calculate $M_{j, \omega}^{t}$ is the sum of all potential customer demands which can be served from $j \in J_{\omega}$ on date $t$, divided by the vehicles capacity:

$$
M_{j, \omega}^{t}=\left\lceil\frac{\sum_{i \in I_{d}, d \in D_{j}, j \in J_{\omega}} q_{i}^{t}}{Q_{\omega}}\right\rceil .
$$

In this case study, we can note that these constraints are useful to avoid producing solutions with FTL routes using intermediate RDC which is closed.

The model can be enriched by two additional constraints deriving from professional practices and decision makers preferences. First, suppliers can specify mutual exclusion inequalities of the form $y_{j_{1}}+y_{j_{2}} \leq 1$ between two competing RDCs $j_{1}$ and $j_{2}$. Second, the service region formed by each open RDC and its associated departments has to be connected and well-shaped. We introduce connectivity inequalities inspired by Rossi et al. [4].

\section{Experiments and case study}

In this section, we present numerical experiments on a real-life case study. All models have been coded in Java using the solver CPLEX 12.6. Tests were run on a PC Intel Core i7-3537 processor 2.0 Ghz with 8 Gb of memory under the System Windows 8.0.

The collaborative distribution network considered includes 4 suppliers, 1 CDC, 29 potential RDC locations, 67 FTL routes. We dispose of an exhaustive recording of one complete year of activity representing 42886 shipments to 3640 customers and 99 shipping dates.

We run the model without any inequality to calculate the optimal locations on one complete year. Several solutions are compared, with the number of RDCs $p$ ranging from 6 to 12. Each instance is solved with a computing time limit of 2 hours. Results are illustrated in Figure 3, which represents the evaluation of total cost and gaps when $p$ increases from 6 to 12. On the left, the horizontal axis represents the relative cost $z_{p}$ for each value of $p$ compared to the cost when $p$ is 6 , computed as $\left(z_{p}-z_{6}\right) / z_{6} \times 100$. We can notice that the cost $z_{p}$ reduces continuously when $p$ increases. The main reason is that $c_{j}$ is set to 0 . On the right, the vertical axis represents the corresponding 

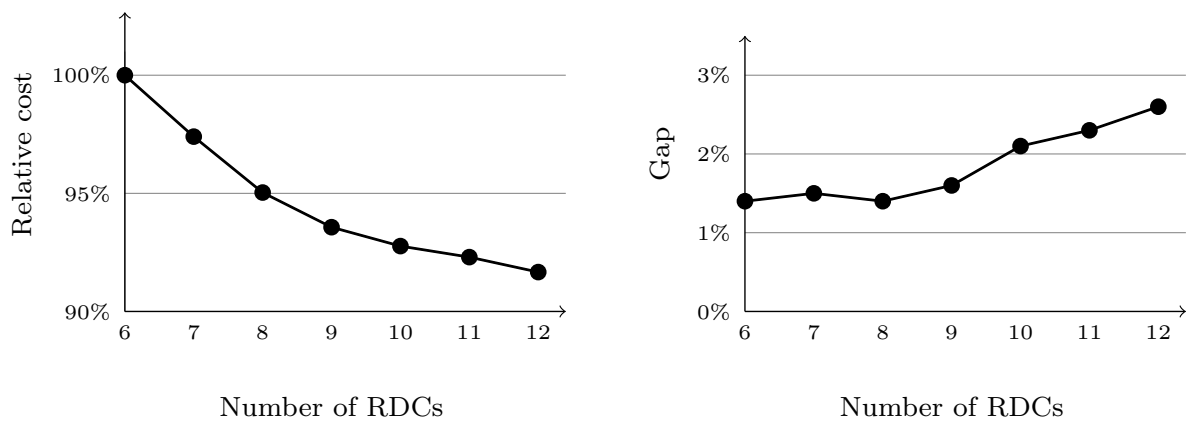

Figure 3. Evaluation of costs and gaps with different value of $p$

relative gaps between the cost of solution $z_{p}$ and the lower bound $z_{l b}$ provided by the solver, computed as $\left(z_{p}-z_{l b}\right) / z_{l b} \times 100$. When $p$ increases, solving the problem becomes more difficult.

In this paper we addressed the optimal location of distribution centers in a collaborative supply chain, considering FTL and LTL shipment costs. Further research includes load plan optimization at each period in the logistics network resulting from this study.

\section{References}

[1] B. K. Bahinipati, A. Kanda, and S. G. Deshmukh. Horizontal collaboration in semiconductor manufacturing industry supply chain: An evaluation of collaboration intensity index. Computers $\mathcal{E}$ Industrial Engineering, 57(3):880895, 2009.

[2] H. M. le Blanc, F. Cruijssen, H. A. Fleuren, and M. B. M. de Koster. Factory gate pricing: An analysis of the dutch retail distribution. European Journal of Operational Research, 174(3):1950-1967, 2006.

[3] M. T. Melo, S. Nickel, and F. Saldanha da Gama. Facility location and supply chain management - A review. European Journal of Operational Research, 196(2):401-412, 2009.

[4] A. Rossi, A. Aubry, and M. Jacomino. Connectivity-and-hop-constrained design of electricity distribution networks. European Journal of Operational Research, 218(1):48-57, 2012. 